\title{
Les vendeurs de maladies
}

Jean Martin

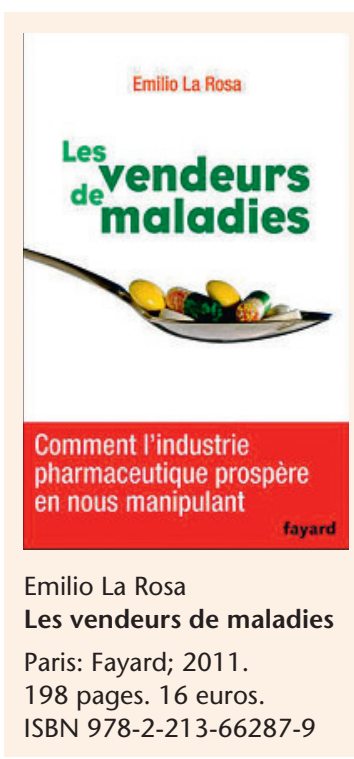

jean.martin@saez.ch
Le Dr La Rosa est médecin et docteur en anthropologie et écologie humaine; il est aussi membre du Comité international de bioéthique de l'Unesco. Il aborde dans cet ouvrage, de manière aisément compréhensible, plusieurs des défis actuels liés aux rapports entre l'industrie pharmaceutique, les autres secteurs du système de santé, notamment les professionnels et académiques, et la santé des personnes. On peut rappeler à ce propos le fait singulier, et problématique, que dans la plupart des pays occidentaux cette industrie, privée et à but lucratif, est le seul grand partenaire du domaine de la santé où il n'y a pas de représentant de l'intérêt général/public dans les instances qui déterminent les stratégies et prennent les décisions d'investir - ou pas. NB: d'autres secteurs établissements sanitaires par exemple - incluent des éléments à but lucratif mais ces derniers restent minoritaires et les pouvoirs publics y jouent un rôle important [1].

Le «disease mongering» (l'invention de maladies, le colportage de bonnes raisons alléguées de juger les gens malades et de prescrire des médicaments [2]) est l'objet d'analyses et de fortes prises de position sur le plan éthique depuis une vingtaine d'années. On se souvient en particulier de l'article de Marcia Angell sur la vénalité dans la médecine universitaire [3]. Des mesures correctrices et préventives ont été prises mais tout n'est certainement pas résolu.

Dans sa première partie, La Rosa discute ainsi la «création corporative de la maladie» et donne des exemples de redéfinition des affections ou facteurs de risques, promouvant l'augmentation des prescriptions médicamenteuses; «étiquetage diagnostique inutile voire nuisible pour le patient»; «beaucoup d'individus sont enfermés [alors] dans les mailles du diagnostic» a dit le Nuffield Council on Bioethics britannique. Est impliquée toute la question délicate de l'élaboration et de l'adoption de recommandations (guidelines) de bonne pratique qui peuvent conduire à des gains majeurs pour l'industrie - élargissement du marché du médicament. Les épopées très lucratives de la dysfonction érectile, et maintenant de la dysfonction sexuelle féminine, sont de notoriété publique. L'auteur note pertinemment que cette promotion des médicaments passe même, et entre autres, par les associations de malades, soutenues par les firmes. Dans la partie II, il décrit les mécanismes à l'œuvre dans la recherche, la mise sur le marché et le commerce des médicaments. Selon une source officielle, l'industrie consacre en France 25000 euros par an et par médecin à la publicité et à l'activité des visiteurs médicaux. Est présentée la situation de groupes de médicaments: hypolipémiants, psychotropes, antidépresseurs et anxiolytiques, ainsi que les histoires instructives du rofécoxib $\left(\operatorname{Vioxx}^{\circledR}\right)$ et du benfluorex (Mediator $^{\circledR}$ ). La Rosa: «Malgré ces difficultés, tout semble indiquer que l'industrie continuera dans la même voie, surtout dans les pays en développement. Le pouvoir et la surdité vont visiblement de pair.»

La dernière partie traite d'abord de démocratie sanitaire, expression introduite et débattue en France à l'occasion de la loi du 4 mars 2002 sur les droits des malades (dite loi Kouchner). Puis elle aborde les règles liées à la bioéthique, mentionnant notamment la Déclaration universelle sur la bioéthique et les droits de l'homme adoptée en 2005 par l'Unesco, instrument international de valeur. La Rosa consacre utilement une vingtaine de pages aux conflits d'intérêts - question très actuelle à juste titre, à laquelle la corporation médicale a mis du temps à être adéquatement sensibilisée.

En résumé, une publication très pertinente qui réussit à présenter, sous une forme concise et agréable à lire, une information essentielle. Qui montre l'importance de la transparence dans un domaine où, si on a le droit d'y gagner de l'argent, de grands défis éthiques (y compris d'éthique sociale) sont lancés à l'industrie. Est-il encore acceptable qu'y règne le principe que des efforts de recherche sont développés pour l'essentiel seulement là où il y a un marché solvable? Alors que sont mis sur le marché de nombreux médicaments «me-too» (à savoir: moi aussi j'en propose un de plus) pour des affections largement couvertes par ce qui est déjà disponible, de grands fléaux infectieux (paludisme et tuberculose spécialement) restent, par le peu d'attractivité du marché potentiel, sans moyens de lutte ou prévention suffisamment efficaces et accessibles. L'OMS et d'autres déploient à cet égard des efforts qu'on peut saluer et l'industrie dit vouloir faire sa part, mais les progrès ne sont guère rapides. Je recommande la lecture du livre du Dr La Rosa.

\section{Références}

1 Martin J. Research in biomedicine - Is anyone representing/advocating the public interest? European Journal of Public Health. 2001;11:458-9. Aussi: Dans la recherche médicale, personne ne défend l'intérêt public. Le Temps (Genève); 12 janvier 2001.

2 Moynihan R, Heath I, Henry D. Selling sickness: the pharmaceutical industry and disease mongering. BMJ. 2002;324:886-91.

3 Angell M. Is academic medicine for sale? N Engl. J Med. 2000;324:1516-8. 